\title{
THE SYNTAX OF -ER NOMINALS: A MINIMALIST APPROACH
}

\author{
KAZUHIRO MASUTOMI \\ Miyagi Gakuin Women's University*
}

Keywords: -er nominals, Merge, the edge feature, Predication, Modification

\section{Introduction}

The main goal of this paper is to explore syntax of event -er nominals (e.g. destroyer, reviewer, supporter) which refer to the external argument of a base verb, in terms of the Minimalist Program (MP) proposed by Chomsky (2006). $-E r$ nominals are one type of productive deverbal morphology in English, and their syntax and semantics have been explored in many works (e.g. Burzio (1986), Roeper (1987), Rappaport and Levin (1992), van Hout and Roeper (1998), Fujita and Matsumoto (2005)). Specifically, the following contrast seems to show the most interesting property of -er nominals, which is concerned with their external argument realization. The following examples are cited from Rappaport and Levin (1992):

(1) a. destroyer, grinder, writer, learner, observer, defender, devourer ... (transitive)

b. runner, jumper, climber, creeper, cruiser, glider, scooter, speaker ... (unergative)

c. *appearer, *occurrer, *happener, *exister, *dier, *ender ... (unaccusative)

According to Rappaport and Levin, the examples in $(1 \mathrm{a}, \mathrm{b})$ show that -er nominals most systematically correspond to the external argument

* I would like to thank Nobuaki Nishioka and David Farnell for their helpful comments and suggestions. I am also deeply indebted to two anonymous $E L$ reviewers for their invaluable comments on an earlier version of this paper. All remaining errors and inadequacies are of course my own. 
of the base verbs: the External Argument Generalization (EAG) in their term. Therefore, the ungrammaticality of the -er nominals in (1c) can be accounted for by the EAG straightforwardly, since it is generally assumed that unaccusative verbs project no external argument in their syntactic structure.

However, what is important is how we can explain such a generalization in a principled way. In this paper, we will solve this problem by discussing details of the operation of Merge. Concretely, we assume that there are (at least) four types of Merge: External Merge (EM), Internal Merge (IM), Adjunction, and Morphological Merger, on the basis of whether the edge feature (EF) triggers the relevant operation (see Chomsky (2006) for the details of the EF, which indicates that a lexical item can be merged). ${ }^{1}$ We propose that the functional differences between these types of Merge play an important role in accounting for the external argument realization of -er nominals.

In the latter part of this paper, we also consider how the interpretation of -er nominals can be determined at the semantic interface. In the recent minimalist works, it is assumed that syntactic objects are transferred to the interface phase by phase, and that they are interpreted there. Therefore, their meaning must be determined on the basis of their structure. In this respect, Chomsky (2006) and Den Dikken (2006) propose that "Proposition" or "Predication" relationships can be interpretable at the semantic interface. In addition to their proposals, we assume that the adjunction structure can be interpreted at the interface, too, namely, as a "Modification" relationship, and that such an interpretation turns out to be the meaning of -er nominals: 'someone or something that does X.'

The organization of this paper is as follows: in the next section we consider a recent analysis of the external argument realization in -er nominalization. In Section 3, we consider the relation between Merge and the EF. Then, we propose the properties of four types of Merge. In Section 4 , we show our minimalist analysis of the derivation of -er nominals on the basis of the proposal in Section 3. Section 5 elaborates the analysis presented in Section 4, and Section 6 is a conclusion.

\footnotetext{
${ }^{1}$ As argued in what follows, we propose that Adjunction is a kind of Merge in that the operation also combines a syntactic object with another syntactic object.
} 


\section{A Previous Analysis}

In the preceding section, we have already introduced the EAG, which describes the most typical property of -er nominals. In the remainder of this paper, we consider how -er nominals realize their external argument in the course of the derivation within the framework of the MP. First of all, in this section, we have a brief discussion about a previous proposal to this question.

Fujita and Matsumoto (2005) assume that the affix -er as well as the passive morpheme -en "suppresses" (or "absorbs") the subject $\theta$-role (i.e. external argument) of a base verb, on the basis of the parallelism between -er nominalization and (pseudo-)passivization as shown in (1) and (2): both cannot be derived from unaccusative verbs.

(2) a. The dog was kicked by the children. (transitive)

b. The bed was slept in by the Shah. (unergative)

(Fujita and Matsumoto (2005))

c. ${ }^{*}$ The desk was fallen from by the book. (unaccusative)

As shown in (3) below, they assume that the affixes -en and -er suppress the subject $\theta$-role of a base verb during (pseudo-)passivization and -er nominalization.

(3) a. [Subj V Obj] $\Rightarrow$ V-en Subj Obj ((pseudo-)passivization)

b. [Subj V Obj] $\Rightarrow$ V-er Subj Obj (-er nominalization)

(Fujita and Matsumoto (2005: 62))

They account for the ungrammaticality of (1c) and (2c) by combining this assumption with the standard assumption that unaccusative verbs have no subject $\theta$-role. In a nutshell, they argue that the affixes -en and -er cannot suppress the subject $\theta$-role of a base verb in the unaccusative construction; as a result, such a derivation is ungrammatical.

However, we cannot accept their analysis, not only because the process of suppression of the subject $\theta$-role has not been defined explicitly, but also because if the operation of Merge is only available in the minimalist framework, then it must be difficult to explain such a process by Merge. Thus, we propose an alternative analysis in the following discussion.

\section{The Relation between Merge and the Edge Feature}

In this paper, we approach the derivational process of -er nominalization by defining the types of Merge specifically, as already mentioned. In order to do so, we now propose that in the minimalist framework (at least) four 
types of Merge can be assumed theoretically: External Merge (EM), Internal Merge (IM), Adjunction, and Morphological Merger (cf. Embick and Noyer (2001) and Matushansky (2006) among others). Of course, all the types of Merge have a common property: they create a new object $\gamma$ by combining (i.e. "merging") a lexical item $\alpha$ with another lexical item $\beta$. But they are distinct from each other in the following properties:

(4) a. $\alpha_{[\mathrm{EF}]}+\beta \Rightarrow\left[{ }_{\mathrm{aP}} \alpha_{[\mathrm{EF}]} \beta\right]_{\gamma} \quad$ (External Merge)

b. $\alpha_{[\mathrm{EF}]}+\left[\begin{array}{llll}\ldots & \beta & \ldots\end{array}\right]{ }_{[\mathrm{aP}} \beta \alpha_{\mathrm{EEF}}\left[\begin{array}{lll}\ldots & t_{\beta} & \ldots\end{array}\right]_{\gamma}$

(Internal Merge)

c. $\beta+\left[\begin{array}{lllll}{ }_{\alpha \mathrm{P}} & \ldots & \alpha & \ldots & \ldots\end{array}\right] \Rightarrow\left[{ }_{\alpha \mathrm{P}} \beta\left[\begin{array}{lllll}{ }_{\alpha \mathrm{P}} & \ldots & \alpha & \ldots & ]_{\gamma}\end{array}\right.\right.$

(Adjunction) $^{2}$

d. $\left[\ldots\left[{ }_{\alpha \mathrm{P}}-\beta_{\text {Affix }}[\alpha \ldots]\right]\right] \Rightarrow\left[\ldots\left[{ }_{\alpha \mathrm{P}} \alpha-\beta_{\text {Affix }} \ldots\right]\right]_{\gamma}$

(Morphological Merger)

EM and IM, which are most popular types of Merge assumed in the MP, are driven by the EF as shown in $(4 \mathrm{a}, \mathrm{b})$ above. We assume that Adjunction, which is also a structure-building operation, merges $\alpha$ with $\beta$ without the EF to create $\gamma$ in syntax, and that Morphological Merger merges $\alpha$ with $\beta$ without the EF under adjacency in Morphology.

In EM and IM, the EF of $\alpha$ is deleted by merging $\beta$ in the edge of $\alpha$. Specifically, we assume that $\alpha$ is able to have two EFs and create two edges: a left edge and a right edge. Then, $\alpha$ can derive the structure shown below, with $\delta_{1}$ and $\delta_{2}$ in the two edges:

(5) [left edge $\delta_{1}\left[\begin{array}{lll}\alpha_{\text {[EF] }} & \left.\delta_{2} \text { right edge }\right]\end{array}\right]$

In (5), the EFs of $\alpha$ is deleted by merging of $\delta_{1}$ and $\delta_{2}$. If an additional item $\beta$ is merged with the structure shown in (5), where the EFs of $\alpha$ have been deleted, such an operation should trigger Adjunction, since Adjunction needs no EFs by the definition proposed just above.

(6) $\beta+\left[\right.$ [left edge $\delta_{1} \quad\left[\begin{array}{lll}\alpha_{\text {EFF }} & \left.\delta_{2} \text { right edge }\right]\end{array}\right] \Rightarrow\left[{ }_{\alpha \mathrm{P}} \beta\left[{ }_{\alpha \mathrm{P}} \delta_{1}\left[\begin{array}{lll}\alpha & \delta_{2}\end{array}\right]\right]\right.$

Morphological Merger merges $\alpha$ with $\beta$ under adjacency, forming a morphological object (i.e. a word). We propose that Affixation is one such

2 In addition to the structure shown in (4c), the adjunction structure can also be illustrated by the following structure, where $\alpha$ (i.e. $\alpha \mathrm{P}$ ) is adjoined to $\beta$ (i.e. $\beta \mathrm{P}$ ):

(i) $\beta \mathrm{P}+\left[\begin{array}{lllll}\alpha \mathrm{P} & \ldots & \alpha & \ldots\end{array}\right]\left[\begin{array}{l}\beta \mathrm{P} \\ \beta \mathrm{P}\end{array}\left[\begin{array}{lllll}\alpha \mathrm{P} & \ldots & \alpha & \ldots & ]_{\gamma}\end{array}\right.\right.$

We propose that (4c) and (i) illustrate the same operation. Thus, we show one of these structures in (4c) for expository convenience. 
case, as illustrated in (4d) above. ${ }^{3}$ At this point, what is important is that Morphological Merger cannot merge $\alpha$ with $\beta$ in the structure shown in (4c), which illustrates the case of Adjunction, because $\beta$ is not in the edge of $\alpha$, and invisible to the operation. ${ }^{4}$

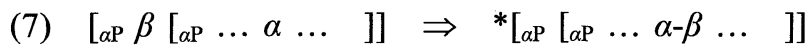

To summarize the discussion above, our proposal is as follows:

(8)

\begin{tabular}{|c|c|c|c|}
\cline { 2 - 4 } \multicolumn{1}{c|}{} & Edge Feature (EF) & Location \\
\hline \multirow{4}{*}{ Merge } & External Merge (EM) & Contained & Syntax \\
\cline { 2 - 4 } & Internal Merge (IM) & Contained & Syntax \\
\cline { 2 - 4 } & Adjunction & Not contained & Syntax \\
\cline { 2 - 4 } & $\begin{array}{c}\text { Morphological } \\
\text { Merger }\end{array}$ & Not contained & Morphology \\
\hline
\end{tabular}

On the basis of such an assumption, we consider the process of -er nominalization concretely in the next section.

\section{The External Argument Realization of $-E R$ Nominals}

We now propose a minimalist analysis of the property of -er nominals under discussion: they need the external argument (position) of a base verb, which has been generalized by the EAG as already discussed. In this section, for the sake of completeness, we compare the derivation of -er nominals with that of other types of derived nominals (i.e. destruction here). The following discussion is based on the parallelism between clauses and noun phrases, more precisely, the NP-shell analysis proposed by

3 We argue that affixation is basically carried out by Head Movement (HM) in syntax. But we also assume that some kinds of affixation (e.g. -er affixation) are achieved in Morphology (see Section 4, for the details).

4 As shown in the following contrast, it has been assumed that the adjunction position is not visible to the operation of Morphological Merger (or Affix Hopping).

(i) [Tr [T -es] [NegP $n o t$ Neg [vp V ... (cf. *John not kisses Mary/John doesn't kiss Mary)

(ii) $[\mathrm{TP}[\mathrm{T}$-es] [vp often $[\mathrm{vp} \mathrm{V} \ldots$ (cf. John often kisses Mary.)

The behavior of the adverb in (ii) indicates that the adjunction position does not undergo the operation, and it does not block the operation, either, in contrast to the specifier position. 
Radford (2004), which proposes that noun phrases are composed of $n \mathrm{P}$ and NP.

First, we assume that the derivation of the enemy's destruction of the city can be schematically illustrated as follows (but the irrelevant parts are ignored in the following discussion):

Head Movement

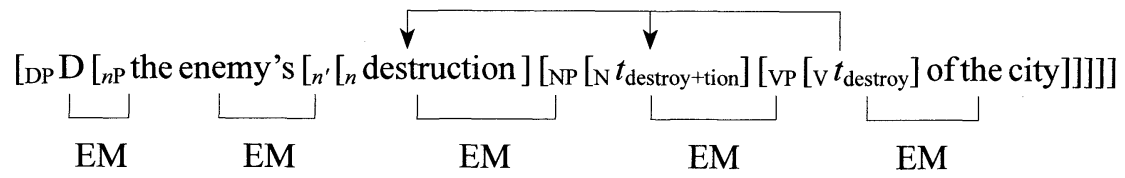

We propose that the syntactic structure of the enemy's destruction of the city is built by EM and the Head Movement (HM) of the verbal root destroy into $n$, which proceeds via $\mathrm{N}$ (i.e. the nominalizing affix -tion), as shown just above (see Radford (2004) and Masutomi (2006), among others, for other motivations for proposing the minimalist analysis of derivational morphology). ${ }^{5}$ In this paper, following Chomsky (2006) and the framework of the Distributed Morphology, we assume that each category is determined by the combination of a lexical Root and a light head (e.g. $v+\mathrm{V}_{\text {Root }}=$ Verb, $n+\mathrm{N}_{\text {Root }}=$ Noun). Then, we propose that $\mathrm{V}_{\text {Root }}+\mathrm{N}_{\text {Root }}+n$ complex shows a category-changing process. Therefore, $[\mathrm{v}$ destroy $]+\left[{ }_{\mathrm{N}}-\right.$ tion $]+n$ complex shown in (9), which stands for destruction, is considered to be a deverbal noun.

We now consider the derivation of -er nominals, for example, the destroyer of the city. On the basis of the derivation illustrated in (9), the intermediate step of the relevant derivation should be as follows:

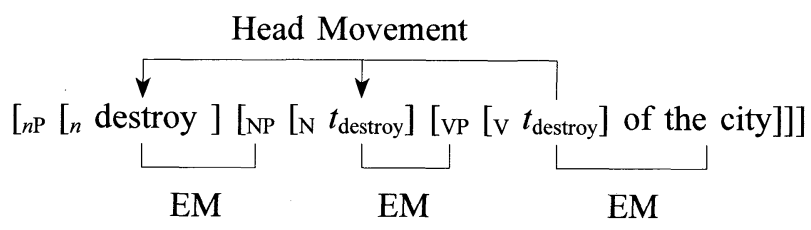

In (10), as in the case of (9), the relevant structure is built by EM and HM. However, the affix -er has not been introduced into the derivation yet in contrast to the affix -tion in (9), where the affix is generated in the head of NP (i.e. [N -tion]). We propose that the affix -er is not a categorychanging affix, since it does not necessarily change the category of a base,

\footnotetext{
5 Similarly, it could be possible to define HM as one of the types of Merge. However, for reasons of space, we cannot address the issue here (see also Matushansky (2006)).
} 
as shown in (11b):

(11) a. [V-er $]_{\mathrm{N}}$ type: destroyer, painter, reader, runner, swimmer, dancer ...

b. $[\mathrm{N}-\mathrm{er}]_{\mathrm{N}}$ type: philosopher, astronomer, Londoner, villager, pensioner ...

Thus, we assume that the affix -er does not occur in the $\mathrm{N}$. Nevertheless, in (10) the head $\mathrm{N}$, which is null in this case, should be merged with the VP on the basis of the NP-shell analysis (see also footnote 7). Therefore, we argue that the affix $-e r$ is merged with the $n \mathrm{P}$ in (10) above by EM, so deriving the following structure:

(12) $\left[{ }_{n \mathrm{P}}-\operatorname{er}\left[n^{\prime}\left[{ }_{n}\right.\right.\right.$ destroy $]\left[\mathrm{NP}\left[\mathrm{N} t_{\text {destroy }}\right]\left[\mathrm{VP}\left[\mathrm{v} t_{\text {destroy }}\right]\right.\right.$ of the city]]]] Consequently, in (12), the affix -er is merged in the left edge of $n$ (i.e. "the external argument position"), deleting the EF of $n$. On the basis of the structural parallelism between clauses and noun phrases, we assume that the external argument is merged in the left edge of $n$, because this position corresponds to the left edge (i.e. specifier) of $v \mathrm{P}$, which is usually considered to be the external argument position in the clausal structure. ${ }^{6}$ In the next step (precisely in Morphology), the affix -er is merged with destroy by Morpho-

${ }^{6}$ For the sake of completeness, consider the following derivation, where one might think that the city and this article are base-generated in the left edge of $n \mathrm{P}$ :

(i) the city's destruction

(ii) this article's reviewer

First, (i) is an example of passive nominals, for which we assume the following derivation:

(iii) [DP the city's D ${ }_{n \mathrm{P}}\left[{ }_{n}\right.$ destruction $\left.\left.]\left[{ }_{\mathrm{NP}}\left[\mathrm{N} t_{\text {destroy+tion }}\right]\left[\mathrm{vP}\left[\mathrm{v} t_{\text {destroy }}\right] t_{\text {the city }}\right]\right]\right]\right]$

In (iii), the head $\mathrm{D}$ agrees with the city, which is base-generated as the object of destroy, and the city moves to the left edge of $\mathrm{D}$, deleting the $\mathrm{EF}$ of the $\mathrm{D}$.

For the example (ii), we assume another derivation, because it cannot be considered to be a passive nominal as shown in the following contrast:

(iv) a. the city's destruction by the enemy

b. *this article's reviewer by the teacher

Therefore, we assume that the example (ii) is derived as follows:

(v) [DP this article's D [ ${ }_{n \mathrm{P}}-\mathrm{er}\left[{ }_{n}^{\prime}\left[{ }_{n}\right.\right.$ review ] [NP $\left[\mathrm{N} t_{\text {review }}\right]\left[\mathrm{VP}\left[\mathrm{v} t_{\text {review }}\right]\right.$ (THEME)]]]]] In (v), the possessive form the article's is base-generated in the left edge of D and modifies the $n \mathrm{P}$, while -er is the external argument and THEME is the implicit thematic role proposed by Roeper (1987). Therefore, we can argue that $-e r$ is the external argument of -er nominals in examples such as (ii), too. 
logical Merger, and derives destroyer successfully., 8

Interestingly, these processes of the derivation account for the statement of the EAG in a principled way. Specifically, if the affix -er is not merged in the left edge of $n$ (i.e. the external argument position) by the EF in contrast to (12), then it must be adjoined to the $n \mathrm{P}$ in (10), so deriving the following structure:

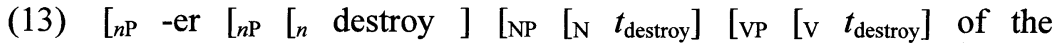
city]]]] (Adjunction)

However, Morphological Merger cannot be applied to such a structure, because the affix -er, which is in the adjunction position, is not visible to the operation, as already discussed. As a result, the affix -er cannot satisfy its affixal requirement, so crashing the derivation. Therefore, we can explain why -er nominals must be related to the external argument (position) of a base verb, in terms of the mechanisms of Merge.

At the same time, such an analysis can predict the asymmetry shown in (1) above: -er nominals are derived from transitive and unergative verbs,

7 Unfortunately, for reasons of space, we cannot discuss the constraint on the realization of internal arguments of -er nominals. In this respect, however, we agree to Roeper's (1987) proposal. He argues that -er entails [AGENT, THEME] but not other thematic roles. Therefore, the example shown in (i) is grammatical, because the thematic grid of -er matches the thematic grid of the base verb driver (i.e. [AGENT, THEME]).

(i) the driver of the truck

(ii) *a speaker to him, *a hoper to leave, *the putter of men on the moon

The ungrammaticality of the examples in (ii) can be explained in terms of the thematic mismatch between -er and the base verbs. The same explanation is also available in our framework on the assumption that the head $\mathrm{N}$ of $-e r$ nominals has the relevant thematic grid (i.e. [AGENT, THEME]). These points also show that the null head $\mathrm{N}$ in (10) or (12) must be present in the derivation of -er nominals in terms of the internal argument realization.

${ }^{8}$ For the sake of completeness, consider the following derivation, where another DP is merged in the external argument position, instead of -er.

(i) $\left[{ }_{n \mathrm{P}}\right.$ the enemy $\left[{ }_{n^{\prime}}\left[{ }_{n}\right.\right.$ destroy $]\left[{ }_{\mathrm{NP}}\left[\mathrm{N} t_{\text {destroy }}\right]\left[\mathrm{vP}\left[\mathrm{v} t_{\text {destroy }}\right]\right.\right.$ of the city] $\left.\left.]\right]\right]$ (cf. (12)) (cf. *the enemy's destroy of the city)

We propose that such a derivation is ruled out in terms of the following lexical selectional property between the $\mathrm{N}$ and -er:

(ii) $\mathrm{N}$ which has [AGENT, THEME] selects $-e r$ as its external argument.

Roeper (1987) argues that -er, which is probably the head N, has the two obligatory thematic roles AGENT and THEME (see also footnote 7). At this point, if we separate -er from the head position (or its thematic roles), then we could translate Roeper's (1987) proposal by the selectional property as shown in (ii). If this argument is on the right track, the enemy cannot be selected as the external argument of -er nominals in (i). As a result, we can rule out the relevant derivation successfully. 
and not from unaccusative verbs. We can predict each structure of -er nominals derived from these verbs, on the basis of the discussion here and the standard assumption shown in (14). Concretely, such structures are shown in (15), where (15c) shows the same type as we have already discussed in (13):

(14) a. [vP external argument $\left[v^{\prime} v[\mathrm{vP} V\right.$ internal argument $\left.\left.]\right]\right]$

(Transitive Verb)

b. [ ${ }_{v \mathrm{P}}$ external argument $\left.\left[v^{\prime} v[\mathrm{vP} \mathrm{V}]\right]\right] \quad$ (Unergative Verb)

c. $\left[{ }_{\nu \mathrm{P}} v[\mathrm{vP} \mathrm{V}\right.$ internal argument]] (Unaccusative Verb)

a. $\left[{ }_{n \mathrm{P}}-\mathrm{er}\left[n^{\prime} n[\mathrm{NP} \mathrm{N}[\mathrm{VP} \mathrm{V}\right.\right.$ internal argument]]]]

(Transitive $-E R$ Nominals)

b. $\quad\left[n \mathrm{P}-\mathrm{er}\left[{ }_{n^{\prime}} n[\mathrm{NP} \mathrm{N}[\mathrm{vP} \mathrm{V}]]\right]\right]$ (Unergative -ER Nominals) ${ }^{9}$

c. $\left[{ }_{n \mathrm{P}}-\mathrm{er}\left[{ }_{n \mathrm{P}} n\left[{ }_{\mathrm{NP}} \mathrm{N}[\mathrm{VP} \mathrm{V}\right.\right.\right.$ internal argument]]]]

(Unaccusative $-E R$ Nominals)

Traditionally, an external argument position is assumed in transitive and unergative constructions, as shown in $(14 a, b)$. Thus, it is also possible to assume such a position (i.e. the left edge of $n$ ) in -er nominals as shown in $(15 \mathrm{a}, \mathrm{b})$, because the $n$ can create the two edges as already discussed in Section 3. As a result, affix -er can be merged there, and we can predict the grammaticality of -er nominals derived from these verbs correctly (see also the discussion in (12)). In contrast, in the case of unaccusative verbs, the relevant position is not assumed traditionally, as shown in (14c). Accordingly, in this case, the $n$ in $(15 \mathrm{c})$ does not create the left edge. ${ }^{10}$ Thus, the affix -er must be adjoined to the $n \mathrm{P}$ as already discussed. In such a structure, Morphological Merger cannot apply to the relevant elements (i.e. the affix -er and V-N-n complex), so that -er affixation cannot be completed. As a result, -er nominals cannot be derived from unaccusative verbs.

In this way, the analysis proposed here can predict the grammaticality of -er nominals in terms of the properties of Merge. In the next section, we consider the relation between the derivational structure as shown in (12) and

9 The derivation in (15b) is compatible with the explanation in footnote 8 , because unergative verbs have a complement, which is incorporated into (or conflated with) V, on the basis of Hale and Keyser's (2002) proposal. Thus, we could argue that the $\mathrm{N}$ in (15b) matches the $\mathrm{V}$ with respect to their thematic roles (i.e. [AGENT, THEME]).

${ }^{10}$ In our framework, this means that the $n$ has only one EF. In this sense, we may argue that the number of the EFs is constrained by the argument structure of a base verb. However, we cannot address this issue here for reasons of space. 
its interpretation.

\section{The Interpretation of $-E R$ Nominals}

We have discussed the derivation of -er nominals so far. In this section, we consider how they are interpreted in the semantic interface, focusing on the relation between syntactic structure and semantic interpretation. In the recent minimalist framework, such a relation can be considered as follows:

(16) Syntactic configuration transferred to the interface (i.e. a phase) must be semantically and phonologically independent (i.e. "interpretable") at the interface.

In this respect, Den Dikken (2006) proposes that such an interpretable configuration is a "predication relationship," and that all predication relationships are syntactically represented as shown in (17), where RELATOR (R) mediates the relationship:

(17) $\left[{ }_{R P} X P\right.$ [ $R^{\prime}$ R YP] $]$

(Den Dikken (2006: 11))

If so, the following structures, where $\mathrm{R}=n$, should also be interpreted as a predication relationship (with irrelevant parts omitted in (18)):

a. [ ${ }_{n \mathrm{P}}$ the enemy's $\left[{ }_{n}^{\prime}\left[{ }_{n}\right.\right.$ destruction $][\mathrm{NP} \ldots$ of the city $\left.\left.]\right]\right]$

b. $\left[{ }_{n \mathrm{P}}-\operatorname{er}\left[{ }_{n^{\prime}}\left[{ }_{n}\right.\right.\right.$ destroy $][\mathrm{NP} \ldots$ of the city $\left.\left.]\right]\right]$ (cf. (12))

Actually, as we expect, the interpretation of (18a) refers to the event or process that the enemy destroyed the city. Thus, the predication relationship (i.e. subject-predicate relationship) between the enemy's and its predicate destruction of the city is established at the $n \mathrm{P}$. On the other hand, the interpretation of $(18 \mathrm{~b})$ refers to someone or something that destroyed the city. Unexpectedly, such an interpretation does not express a predication relationship. More precisely, it seems to express a "modification relationship" between someone or something and the event denoted by the $n \mathrm{P}$. Therefore, if Den Dikken's assumption is on the right track, this fact shows that the syntactic structure of -er nominals shown in (18b), which we have proposed in the previous section, needs to be elaborated in terms of its interpretation. $^{11}$

So, we assume that the modification relationship is syntactically rep-

11 Den Dikken (2006) proposes that modification relationship is also represented by the structure in (17). However, such an analysis cannot account for the semantic difference between (18a) and (18b). Thus, in this regard, we do not support his analysis. 
resented by an adjunction structure, as is usually assumed in relative clauses. Concretely, on the assumption proposed here, we assume that the relevant structure of -er nominals can be illustrated as follows (see also footnote 2, for the adjunction structure in (19)):

(19) [DP [DP SOMEONE/SOMETHING] ${ }_{n \mathrm{P}}$-er $\left[{ }_{n^{\prime}}\left[{ }_{n}\right.\right.$ destroy $][\mathrm{NP} \ldots$ of the city]]]]

In (19), the $n \mathrm{P}$ is adjoined to a null DP (e.g. [DP SOMEONE/SOMETHING]), so deriving the adjunction structure. As shown in such a structure, we assume that there also exists some element such as the head of relative clauses in -er nominalization on the basis of the similarity between -er nominals and relative clauses, which we have just mentioned. However, in the relevant structure, there is not such an overt element. Therefore, in (19) it seems plausible to assume the null DP as such an element. In addition, if the null DP is selected in the relevant derivation, it seems possible to introduce the null DP into the syntactic structure as in the case of relative clauses. When this syntactic configuration is transferred to the semantic interface, it should be interpreted as a modification relationship there: 'someone or something that destroyed the city.' From these points and the discussion so far, we conclude that the precise syntactic structure of -er nominals (e.g. the destroyer of the city) is illustrated as follows, with the subsequent Merge of the determiner the with the DP in (19):

(20) [DP the [DP [DP SOMEONE/SOMETHING] $\left[{ }_{n \mathrm{P}}-\operatorname{er}\left[{ }_{n}^{\prime}\left[{ }_{n}\right.\right.\right.$ destroy $]$

Morphological Merger

[NP [N $\left.t_{\text {destroy }}\right]\left[\mathrm{vP}\left[\mathrm{v} t_{\text {destroy }}\right]\right.$ of the city]]]]]]

As a consequence of the discussion here, we finally propose the following relationship between syntax and semantics:

Syntax

subject-predicate relationship

adjunction relationship

$\begin{array}{cc} & \text { Semantics } \\ \Rightarrow & \text { Predication } \\ \Rightarrow & \text { Modification }\end{array}$

\section{Conclusion}

In this paper, we have considered the syntax of -er nominals, focusing on the empirical fact described by the EAG. As a result of the discussion here, we have shown that the derivational processes of -er nominals (and other types of derived nominals) can be uniformly analyzed in terms of Merge, the (only) operation of the MP. At the same time, we have argued that the operation of Merge should be defined more precisely in order to 
make such an analysis work. Furthermore, we have considered the relation between syntax and semantics in terms of the types of syntactic configuration.

The results of this paper should have some implications for the minimalist analysis of derivational morphology.

\section{REFERENCES}

Burzio, Luigi (1986) Italian Syntax: A Government-Binding Approach, Reidel, Dordrecht.

Chomsky, Noam (2006) "Approaching UG from Below," ms., MIT.

Den Dikken, Marcel (2006) Relators and Linkers: The Syntax of Predication, Predicate Inversion, and Copulas, MIT Press, Cambridge, MA.

Embick, David and Rolf Noyer (2001) "Movement Operations after Syntax," Linguistic Inquiry 32, 555-595.

Fujita, Koji and Masumi Matsumoto (2005) Goihanchu (I): Doshi (Lexical Category (I): Verb), Kenkyusha, Tokyo.

Hale, Ken and Samuel Jay Keyser (2002) Prolegomenon to a Theory of Argument Structure, MIT Press, Cambridge, MA.

Masutomi, Kazuhiro (2006) "Judomeishiku no Hasei to Bunposei ni tsuite (On the Derivation and Grammaticality of Passive Nominals)," JELS 23, 140-149.

Matushansky, Ora (2006) "Head Movement in Linguistic Theory," Linguistic Inquiry 37, 69-109.

Radford, Andrew (2004) Minimalist Syntax: Exploring the Structure of English, Cambridge University Press, Cambridge.

Rappaport Hovav, Malka and Beth Levin (1992) “-Er Nominals: Implications for the Theory of Argument Structure," Syntax and the Lexicon, Syntax and Semantics 26, ed. by Tim Stowell and Eric Wehrli, 127-153, Academic Press, San Diego.

Roeper, Thomas (1987) "Implicit Arguments and the Head-Complement Relation," Linguistic Inquiry 18, 267-310.

van Hout, Angeliek and Thomas Roeper (1998) "Events and Aspectual Structure in Derivational Morphology," MIT Working Papers in Linguistics 32, 175-200.

Faculty of Liberal Arts

Miyagi Gakuin Women's University

9-1-1 Sakuragaoka, Aoba-ku

Sendai 981-8557

e-mail: mstmkzhr@yahoo.co.jp 